\title{
Effective Hamiltonian for an extended Kondo-lattice model and a possible origin of charge ordering in half-doped manganites
}

\author{
Shun-Qing Shen and Z. D. Wang* \\ Department of Physics, The University of Hong Kong, Pokfulam Road, Hong Kong, China
}

(Received 21 October 1998; revised manuscript received 6 January 1999)

\begin{abstract}
An effective Hamiltonian is derived in the case of the strong Hund coupling and on-site Coulomb interaction by means of a projective perturbation approach. A physical mechanism for charge ordering in half-doped manganites $\left(R_{0.5} X_{0.5} \mathrm{MnO}_{3}\right)$ is proposed. The virtual process of electron hopping results in antiferromagnetic superexchange and a repulsive interaction, which may drive electrons to form a Wigner lattice. The phase diagram of the ground state of the model is presented at half doping. In the case of formation of a Wigner lattice, we prove that spins of electrons are aligned ferromagnetically as well as that the localized spin background is antiferromagnetic. The influence of the on-site Coulomb interaction is also discussed.

[S0163-1829(99)01621-5]
\end{abstract}

\section{INTRODUCTION}

The family of doped manganites, $R_{1-x} X_{x} \mathrm{MnO}_{3}$ (where $R=\mathrm{La}, \mathrm{Pr}, \mathrm{Nd} ; X=\mathrm{Sr}, \mathrm{Ca}, \mathrm{Ba}, \mathrm{Pb}$ ), has renewed both experimental and theoretical interests due to the colossal magnetoresistance and its potential technological application to magnetic storage devices. Apart from their unusual magnetic transport properties, experimental observations of a series of charge, magnetic, and orbital ordering states in a wide range of dopant also stimulate extensive theoretical curiosities. Early theoretical studies of manganites concentrated their effort on the existence of metallic ferromagnetism. From the so-called "double-exchange" (DE) model, ${ }^{1}$ in which the mobility of itinerant electrons forces the localized spins to align ferromagnetically, one can understand qualitatively the relation of transport and magnetism. However, the rich experimental phase diagrams are far beyond the DE model. For example, according to the DE model, itinerant electrons have the lowest kinetic energy in a tight-binding model, and should be driven to form a more stable ferromagnetic phase when the system is half doped, i.e., $x=0.5$. On the contrary, it is insulating rather than metallic ferromagnetic at a low temperature as expected theoretically. Furthermore, a chargeordered state was observed, which is characterized by an alternating $\mathrm{Mn}^{3+}$ and $\mathrm{Mn}^{4+}$ ions arrangement in the real space. ${ }^{2}$ Usually when the repulsive interaction between charge carriers dominates over the kinetic energy the charge carriers are driven to form a Wigner lattice. It has been shown experimentally that the charge ordering is sensitive to an applied magnetic field at low temperatures: resistance of a sample may decrease in several order of magnitude and the charge ordering disappears at a low temperature, ${ }^{3}$ which implies that the repulsive interaction should have a close relation to the spin background. Although there have been extensive theoretical efforts on anomalous magnetic properties, ${ }^{4}$ a comprehensive understanding on the physical origin of ordered states and their relations to the transport properties are still awaited.

To explore electronic origin of these phenomena, we try to establish a more unified picture to understand the physics starting from an electronic model, which has been used to investigate the magnetic properties of the system extensively. We derive an effective Hamiltonian in the case of the strong on-site Coulomb interaction and Hund coupling by means of a projective perturbation approach. It is found that the virtual process of electron hopping produces an antiferromagnetic superexchange coupling between localized spins and a repulsive interaction between itinerant electrons. The antiferromagnetic correlation will enhance the repulsive interaction and suppress the mobility of electrons. In the halfdoped case, i.e., $x=0.5$, relatively strong repulsion will drive electrons to form a Wigner lattice. In the case of the Wigner lattice, we prove that the electrons are fully saturated while the localized spins form an antiferromagnetic background. Strictly speaking, the ground state possesses both anti- and ferromagnetic, i.e., ferrimagnetic long-range orders.

\section{EFFECTIVE HAMILTONIAN}

The electronic model for doped manganites studied in this paper is defined as ${ }^{5}$

$$
\begin{aligned}
H= & -t \sum_{\langle i j\rangle, \sigma} c_{i, \sigma}^{\dagger} c_{j, \sigma}+U \sum_{i} n_{i, \uparrow} n_{i, \downarrow} \\
& -J_{H} \sum_{i} \mathbf{S}_{i} \cdot \mathbf{S}_{i c}+J_{A F} \sum_{\langle i j\rangle} \mathbf{S}_{i} \cdot \mathbf{S}_{j},
\end{aligned}
$$

where $c_{i, \sigma}^{\dagger}$ and $c_{i, \sigma}$ are the creation and annihilation operators for $e_{g}$ electron at site $i$ with spin $\sigma(=\uparrow, \downarrow)$, respectively. $\langle i j\rangle$ runs over all nearest-neighbor pairs of lattice sites. $\mathbf{S}_{i c}=\Sigma_{\sigma, \sigma^{\prime}} \sigma_{\sigma \sigma^{\prime}} c_{i, \sigma}^{\dagger} c_{i, \sigma^{\prime}} / 2$ and $\sigma$ are the Pauli matrices. $\mathbf{S}_{i}$ is the spin operator of three $t_{2 g}$ electrons with the maximal value $3 / 2 . J_{H}>0$ is the Hund coupling between the $e_{g}$ and $t_{2 g}$ electrons. The antiferromagnetic coupling originates from the virtual process of superexchange of $t_{2 g}$ electrons. In reality, the $e_{g}$ orbital is doubly degenerated. For the sake of simplicity, we only consider one orbital per site, which amounts to assuming a static Jahn-Teller distortion and strong on-site interactions (relative to kinetic energy). 


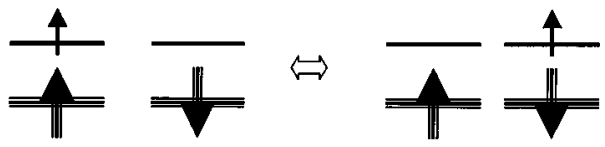

(a)

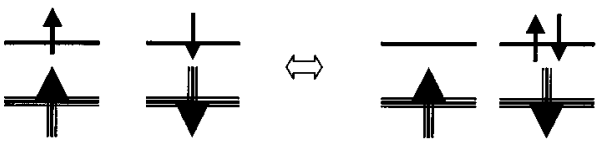

(b)

FIG. 1. Two virtual processes of electron hopping in the restricted Hilbert space which favors antiferromagnetic correlation between neighboring sites. On the left side are the initial states, and on the right side are the mediate states. The process (a) leads to an effective attraction between electron and hole, and the process (b) leads to an effective attraction between electrons.

Usually the Hund coupling in the doped manganites is very strong, i.e., $J_{H} S \gg t$. Large $J_{H} S$ suggests that most electrons form spin $S+1 / 2$ states with the localized spins on the same sites, which makes it appropriate to utilize the projective perturbation technique to investigate the low-energy physics of the Hamiltonian (1). The effect of finite and large $J_{H} S$ can be regarded as the perturbation correction to the case of infinite $J_{H}$, which is described by a quantum doubleexchange model. ${ }^{6}$ Up to the second-order perturbation correction, there are two types of the virtual processes which contribute to the low-energy physics [see Fig. 1]. In Fig. 1(a), an electron hops from one site to one of the nearestneighbor empty sites to form a spin $S-1 / 2$ state and then hops backward. The intermediate state has a higher energy $\Delta E_{a}=J_{H}(S+1 / 2)$ than the initial state. In Fig. 1(b), one electron hops from one site to one of the singly occupied sites and then backward. The intermediate state has a higher energy $\Delta E_{b}=J_{H} S+U$ than that of the initial state. Hence, by using a projective perturbation approach, ${ }^{8,9}$ the effective Hamiltonian is written as ${ }^{7}$

$$
\begin{aligned}
H_{\mathrm{eff}}= & -t \sum_{i j, \sigma} \bar{c}_{i, \sigma}^{\dagger} \bar{c}_{j, \sigma}+J_{\mathrm{AF}} \sum_{\langle i j\rangle} \overline{\mathbf{S}}_{i} \cdot \overline{\mathbf{S}}_{j} \\
& +\frac{2 S t^{2}}{J_{H}(2 S+1)^{2}} \sum_{i j}\left(\frac{\mathbf{S}_{i}}{S} \cdot \frac{\widetilde{\mathbf{S}}_{j}}{S+1 / 2}-1\right) P_{i h} P_{j s}^{+} \\
& +\frac{t^{2}}{J_{H} S+U} \sum_{i j}\left(\frac{\widetilde{\mathbf{S}}_{i}}{S+1 / 2} \cdot \frac{\widetilde{\mathbf{S}}_{j}}{S+1 / 2}-1\right) P_{i s}^{+} P_{j s}^{+},
\end{aligned}
$$

where $\overline{\mathbf{S}}_{i}=\mathbf{S}_{i} P_{i h}+2 S \widetilde{\mathbf{S}}_{i} P_{i s}^{+} /(2 S+1)$ and

$$
\bar{c}_{i, \sigma}=\sum_{\sigma^{\prime}} \frac{\mathbf{S}_{i} \cdot \sigma_{\sigma \sigma^{\prime}}+(S+1) \delta_{\sigma \sigma^{\prime}}}{2 S+1}\left(1-n_{i,-\sigma^{\prime}}\right) c_{i, \sigma^{\prime}} .
$$

$\widetilde{\mathbf{S}}_{i}$ is a spin operator with spin $S+1 / 2$, and a combination of spin of electron and localized spin on the same site. $P_{i h}$ and $P_{i s}^{+}$are the projection operators for empty site and single occupancy of spin $S+1 / 2$. The first term in Eq. (2) is the quantum double-exchange model. ${ }^{6,8}$ It enhances ferromagnetic correlation, and may be suppressed if the antiferromagnetic exchange coupling of localized spin is very strong. The second, third, and fourth terms prefer antiferromagnetism to ferromagnetism. The third term describes an attractive particle-hole interaction since the value of the operator before $P_{i h} P_{j s}^{+}$is always nonpositive. In other words, a repulsive interaction between electrons in the restricted space arises when the spin background deviates from a saturated ferromagnetic case.

To simplify the problem, we take the large spin approximation, and keep $J_{H} S=j_{h}$ and $J_{\mathrm{AF}} S^{2}=j_{a f}$. The spin operator is parametrized in polar angle $\theta$ and $\phi$. In the approximation, the Hamiltonian is further reduced to

$$
\begin{aligned}
H_{\mathrm{cl}}= & -t \sum_{i j} c_{i j} \alpha_{i}^{\dagger} \alpha_{j}-2 j_{a f} \sum_{i j} \sin ^{2} \frac{\Theta_{i j}}{2} \\
& +\sum_{i j} 2 \sin ^{2} \frac{\Theta_{i j}}{2}\left(\frac{t^{2}}{2 j_{h}}-\frac{t^{2}}{j_{h}+U}\right) \alpha_{i}^{\dagger} \alpha_{i} \alpha_{j}^{\dagger} \alpha_{j} \\
& -\sum_{i j} \frac{t^{2}}{2 j_{h}} \sin ^{2} \frac{\Theta_{i j}}{2}\left(\alpha_{i}^{\dagger} \alpha_{i}+\alpha_{j}^{\dagger} \alpha_{j}\right),
\end{aligned}
$$

where

$$
\begin{gathered}
c_{i j}=\cos \frac{\theta_{i}}{2} \cos \frac{\theta_{j}}{2}+\sin \frac{\theta_{i}}{2} \sin \frac{\theta_{j}}{2} e^{-i\left(\phi_{i}-\phi_{j}\right)}, \\
\cos \Theta_{i j}=\cos \theta_{i} \cos \theta_{j}+\sin \theta_{i} \sin \theta_{j} \cos \left(\phi_{i}-\phi_{j}\right), \\
\alpha_{i}=\cos \frac{\theta_{i}}{2}\left(1-n_{i, \downarrow}\right) c_{i, \uparrow}+\sin \frac{\theta_{j}}{2}\left(1-n_{i, \uparrow}\right) c_{i, \downarrow} .
\end{gathered}
$$

Physically, $\alpha$ is an electronic operator which is fully polarized along the localized spin on the same site. $\left|c_{i j}\right|$ $=\cos \left(\Theta_{i j} / 2\right)$ and approaches zero when $\Theta_{i j} \rightarrow \pi$. If we neglect the Berry phase in $c_{i j}$, the first term gets back to the classical DE model. Now it is clear that the ferromagnetism is always predominant in the ground state if other terms in the effective Hamiltonian [Eq. (3)] are neglected. The sign of the interaction

$$
V_{i j}=2 \sin ^{2} \frac{\Theta_{i j}}{2} \frac{t^{2}}{2 j_{h}} \frac{U-j_{h}}{U+j_{h}}
$$

is determined by the ratio $j_{h} / U$. If $U$ is less than $j_{h}$, the interaction is attractive, but if $U$ is greater than $j_{h}$, the interaction is repulsive. The attractive or repulsive interaction will lead to different physics. Hence, $U=j_{h}$ is a quantum critical point. The influence of the on-site Coulomb interaction will change qualitatively (not just quantitatively) the physics of the doped manganites, which is usually ignored. In the case of small $U$, the attractive interaction will drive electrons to accumulate to form an electron-rich regime, i.e., the phase separation may occur when the spin background becomes antiferromagnetic. ${ }^{9}$ Monte Carlo simulation by Dagotto et al. ${ }^{10}$ shows that the phase separation occurs in the case of $U=0$. However, the phenomenon was not observed in the case of large $U$. The phase diagram of $U=0$ is also seen in Ref. 11. From our analysis, the attractive interaction originates from the virtual process (b). Due to the double 
occupancy in the intermediate state, an extra energy $U$ costs in the process. When $U$ is sufficiently large, the process (b) will be suppressed and the process (a) becomes predominant. The net interaction between electrons is repulsive. Therefore, the phase separation may occur only if $U<j_{h}$.

\section{ORIGIN OF WIGNER LATTICE}

We are now in the position to discuss the instability to the Wigner lattice. In the doped manganites, the on-site Coulomb interaction is much stronger than the Hund's rule coupling, i.e., $U \gg J_{H} S .{ }^{12}$ In this case, the process (b) in Fig. 1 needs a much higher energy to be excited than the process (a) does. The process (a) dominates over the process (b). The effective interaction is repulsive. Hence we shall focus on the case of strong correlation (i.e., $U \gg J_{H} S$ ). To simplify the problem, we take $U \rightarrow+\infty$ and neglect the term containing $U$ in Eq. (3). A finite and large $U$ will produce minor quantitative (not qualitative) changes of the physics we shall discuss. The ratio of the repulsion to the hopping term $r$ $=\left(t / j_{h}\right) \sin ^{2}\left(\Theta_{i j} / 2\right) / \cos \left(\Theta_{i j} / 2\right)$ depends on not only $t / j_{h}$, which is usually very small, but also the angle of two spins. $r=0$ if $\Theta_{i j}=0$, and $+\infty$ if $\Theta_{i j}=\pi$. In other words, the ratio could become divergent in the antiferromagnetic spin background $\left(\Theta_{i j}=\pi\right)$ even though $t / j_{h}$ is very small. Relatively large ratio will make a state with a uniform density of electrons unstable. To understand the physical origin for the Wigner lattice at $x=0.5$, we first see what happens in the antiferromagnetic background. When all $\Theta_{i j} \rightarrow \pi$, the average energy per bond is $-2 j_{h}$ if the two sites are empty or occupied, and $-\left(2 j_{a f}+t^{2} / j_{h}\right)$ if one site is empty and another one is occupied. The latter has a lower energy. At $x$ $=1 / 2,\left\langle\alpha_{i}^{\dagger} \alpha_{i}\right\rangle=1 / 2$. The average energy per bond is $-\left(2 j_{a f}+t^{2} / 2 j_{h}\right)$ for a state with a uniform density of electrons. If the electrons form a Wigner lattice, i.e., $\left\langle\left(\alpha_{i}^{\dagger} \alpha_{i}\right.\right.$ $\left.-1 / 2)\left(\alpha_{j}^{\dagger} \alpha_{j}-1 / 2\right)\right\rangle=-1 / 4$, the average energy per bond is $-\left(2 j_{a f}+t^{2} / j_{h}\right)$, which is lower than that of the state with a uniform density. Therefore, in the antiferromagnetic background a uniform density state is not stable against the Wigner lattice even for a small $t / j_{h}$. The same conclusion can be reached by means of the random-phase approximation. On the other hand, the formation of the Wigner lattice will also enhance the antiferromagnetic exchange coupling from $-j_{a f}$ to $-\left(j_{a f}+t^{2} / 2 j_{h}\right)$.

The phase diagram of the ground state is determined by the mean-field approach. Several of the features are determined in several limits: for example, the ground state is ferromagnetic at $t / j_{h}=0$ and $j_{a f}=0$. Due to the instability to the Wigner lattice or charge-density wave for finite $t / j_{h}$ and $j_{a f}$ we take $\left\langle\alpha_{i}^{\dagger} \alpha_{i}-1 / 2\right\rangle=\Delta e^{i \mathbf{Q} \cdot \mathbf{r}_{i}}$ where $\mathbf{Q}=(\pi, \pi, \cdots)$ and $\langle\cdots\rangle$ is the ground-state average. We also take $\left\langle c_{i j}\right\rangle$ $=\cos (\Theta / 2)$ and $\left\langle\sin ^{2}\left(\Theta_{i j} / 2\right)\right\rangle=\sin ^{2}(\Theta / 2) .{ }^{13}$ The free energy per bond is

$$
\begin{aligned}
\mathcal{E}(\Delta, \Theta)= & -\int \frac{d k}{(2 \pi)^{d}} \sqrt{\epsilon^{2}(k) \cos ^{2} \frac{\Theta}{2}+4 \frac{t^{4}}{j_{h}^{2}} \sin ^{4} \frac{\Theta}{2} \Delta^{2}} \\
& -\left(j_{a f}+\frac{1}{4} \frac{t^{2}}{j_{h}}\right) \sin ^{2} \frac{\Theta}{2}+\frac{t^{2}}{j_{h}} \sin ^{2} \frac{\Theta}{2} \Delta^{2},
\end{aligned}
$$

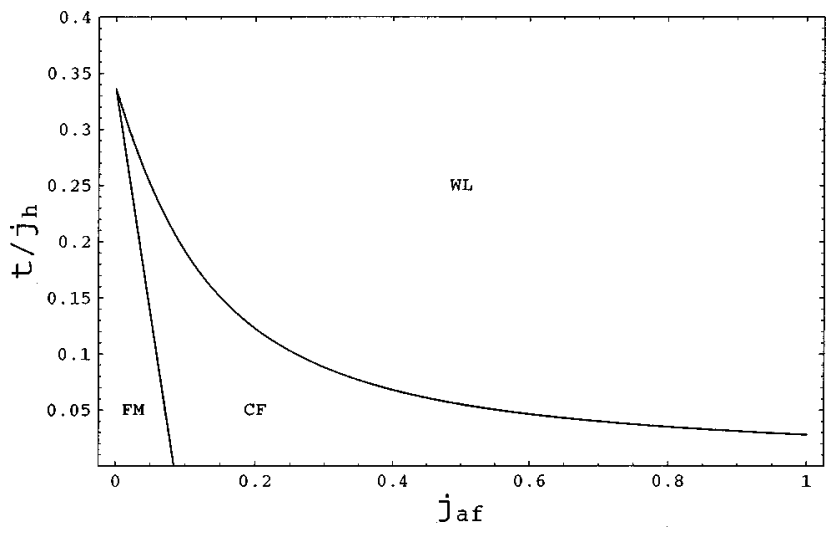

FIG. 2. The phase diagram of the ferromagnetic Kondo lattice model on a cubic lattice $(d=3)$ at $x=1 / 2$.

where $\epsilon(k)=-t\left(\sum_{\alpha=1}^{d} \cos k_{\alpha}\right) / d$ and $d$ is the number of dimension. The integration runs over the reduced Brillouin zone. The phase diagram (Fig. 2) is obtained by minimizing the energy $\mathcal{E}(\Delta, \Theta) . \Delta$ and $\Theta$ are the order parameters for charge and magnetic orderings, respectively. $\Delta=0$ and $\Theta$ $=0$ represents a full ferromagnetic phase, $\Delta=0$ and $\Theta \neq 0$ represents a canted ferromagnetic phase, $\Delta=1 / 2$ and $\Theta=\pi$ represents the Wigner lattice, and $\Delta<1 / 2$ and $\Theta \neq 0$ represents a mixture of charge- and spin-density waves. A full ferromagnetic phase diagram appears at smaller $t / j_{h}$ and $j_{a f}$, which indicates that the double-exchange ferromagnetism is predominant. The Wigner lattice appears at a larger $t / j_{h}$ and $j_{a f}$. The antiferromagnetic coupling originating from the virtual process (a) and superexchange coupling of the localized spins can suppress the double-exchange ferromagnetism completely. A canted ferromagnetic phase is between the two phases. At $j_{a f}=0$, the transition from ferromagnetism to the Wigner lattice occurs at $t^{2} / j_{h}=2 \int d k \epsilon(k) /(2 \pi)^{d}$ which equals $0.63662 t$ for $d=1,0.405282 t$ for $d=2$, and $0.336126 t$ for $d=3$. When the effective potential energy $t / j_{h}$ begins to dominate over the kinetic energy, the ferromagnetic phase is unstable against the Wigner lattice. For a finite $j_{a f}$, a smaller $t / j_{h}$ is required to form a Wigner lattice. However $t / j_{h}$ must be nonzero, even for a large $j_{a f}$. In the double-exchange model, i.e., $j_{h} \rightarrow+\infty$, we do not expect that the Wigner lattice could appear at low temperatures at $x$ $=1 / 2$ unless a strong long-range Coulomb interaction is introduced.

\section{FERRIMAGNETISM AND WIGNER LATTICE}

We go back to Eq. (2) to discuss the magnetic properties of the ground state (or at zero temperature) in the case that the Wigner lattice is formed at $x=1 / 2(1-x$ is the density of electrons). The charge ordering in the manganite is an alternating $\mathrm{Mn}^{3+}$ and $\mathrm{Mn}^{4+}$ arrangement rather than a charge-density modulation, which means $n_{i}=1$ or 0 . A $d$-dimensional hypercubic lattice can be decomposed onto two sublattices $\mathcal{A}$ and $\mathcal{B}$. In the charge-ordering state, suppose that all electrons occupy the sublattice $\mathcal{A}$, then

$$
P_{i h} P_{j s}^{+}= \begin{cases}1, & \text { if } i \in \mathcal{B} \text { and } j \in \mathcal{A} \\ 0, & \text { otherwise }\end{cases}
$$


The first term in Eq. (2) must be suppressed completely as the Wigner lattice is a static real-space pattern, i.e., the hopping processes are forbidden. In this case, the Hamiltonian is reduced to

$$
H_{\mathrm{AF}}=J_{\mathrm{AF}}^{\prime} \sum_{i \in \mathcal{B}, j \in \mathcal{A}}\left(\frac{\mathbf{S}_{i}}{S} \cdot \frac{\widetilde{\mathbf{S}}_{j}}{S+1 / 2}-1\right),
$$

where $J_{\mathrm{AF}}^{\prime}=J_{\mathrm{AF}} S^{2}+2 S t^{2} / J_{H}(2 S+1)^{2}$ and the summation runs over the nearest-neighbor pairs. This is an antiferromagnetic Heisenberg model. The spin on the sublattice $\mathcal{A}$ is $S$ $+1 / 2$ as the electrons on the sites form spin $S+1 / 2$ state with the localized spins, and the spin on the sublattice $\mathcal{B}$ is $S$. According to the Lieb-Mattis theorem, ${ }^{14}$ the ground state of Eq. (7) is unique apart from spin $\mathrm{SU}(2)\left(2 S_{\text {tot }}+1\right)$-fold degeneracy. The total spin of the ground state $S_{\text {tot }}$ is equal to the difference of the maximal total spins of two sublattices. In that case,

$$
S_{\text {tot }}=\frac{N_{e}}{2}
$$

which is also the maximal total spin of electrons $\left(N_{e}\right.$ is the number of electrons). It seems to be that all electrons are saturated fully while the localized spins form a spin singlet state. Furthermore, it is shown rigorously that the groundstate possesses antiferromagnetic long-range order as well as ferromagnetic for any dimension. ${ }^{15}$

\section{DISCUSSION AND SUMMARY}

We wish to point out that, in the case that the Wigner lattice is formed, the magnetic structure established here is unlikely to be in full agreement with all experimental observations. ${ }^{16,17}$ The model discussed here is a simplified theoretical model which has neglected some effects, such as the orbital degeneracy of $e_{g}$ electrons, strong Jahn-Teller effect and lattice distortion. Methodologically, we apply the projective perturbation approach to deal with the model. The strong electron-electron correlations has been successfully taken into account by the projection process. The perturbation process tells us that the effective Hamiltonian should be valid at small $t / j_{h}$, which requires a strong Hund coupling comparing with the hopping integral $t$. In practice, the parameters of the model for doped manganites are roughly estimated as $U \approx 5.5 \mathrm{eV}, \quad J_{H} \approx 0.76 \mathrm{eV}, \quad t \approx 0.41 \mathrm{eV}, \quad J_{\mathrm{AF}}$ $\approx 2.1 \mathrm{meV} .{ }^{12}$ Thus, $U / J_{H} S \approx 4.82$ and $t / J_{H} S \approx 0.359$. For these parameters, the Wigner lattice at low temperatures is stable in the phase diagram in Fig. 2. Therefore, the superexchange process in Fig. 1(a) should play an important role in driving electrons to form the Wigner lattice no matter whether the direct nearest-neighbor Coulomb interaction is strong. It is worth mentioning that the direct Coulomb interaction will always favor forming the Wigner lattice. ${ }^{18}$ If the direct Coulomb interaction is also included in the electronic model, which is not much screened, the stability of the Wigner lattice will be greatly enhanced. Note that the Coulomb interaction is independent of the magnetic structure, and should not be very sensitive to an external magnetic field. The effect of field-induced melting of the Wigner lattice suggests that the physical origin of the state may be closely related to the magnetic structure, which is an essen- tial ingredient of the present theory. In the actual compounds, both the mechanisms should have an important impact on the electronic behaviors. It is unlikely that only one of them is predominant. As for the mean-field approximation, when it is sure that the instability of the Wigner lattice occurs at low temperatures, it is an efficient and powerful tool to determine the phase diagram, although some other physical quantities, such as critical exponents, cannot be obtained accurately. Due to the strong correlations of electrons, we still have a lack of numerical results to verify the present theoretical prediction as this is a discussion on the instability of the Wigner lattice in a model without nearest-neighbor or long-range interactions. When the system deviates from $x$ $=0.5$, the superexchange interaction is still very important to determine the behaviors of electrons. Recently, it was observed that there are paired charge stripes in $\left(\mathrm{La}_{1-x} \mathrm{Ca}_{x}\right) \mathrm{MnO}_{3} \quad(x>0.5) .{ }^{19}$ However, the two pairing stripes of $\mathrm{Mn}^{3+}$ ions are separated by a stripe of $\mathrm{Mn}^{4+}$ ions. This fact suggests the nearest-neighbor interaction should be very strong. Of course, for a comprehensive understanding of the phase diagram, including anisotropic properties of charge and magnetic orderings, we need to take other effects into account.

The role of the Hund's rule coupling in the doped manganites has been emphasized since the double-exchange mechanism was proposed. However, the rich phase diagrams in the doped manganites go beyond the picture. Our theory shows that the on-site Coulomb interaction also has an important impact on the physical properties of the system. In the model we investigate the sign of the effective interaction in Eq. (4), which depends on the ratio of $j_{h} / U$. The repulsive or attractive interaction will lead to quite different physics. In one of our recent papers, ${ }^{9}$ we proposed a mechanism of phase separation based on the attractive interaction caused by the virtual process (a) in Fig. 1, and neglect the on-site interaction $U$. The phase separation can occur in the high and low doping regions. As the mechanism of the phase separation is completely opposite to the mechanism of the Wigner lattice we discuss in this paper, we have to address the issue which occurs for the doped manganites. From the estimation of the model parameters for the actual compound $U / j_{h}$ $\approx 4.82$. Thus, the effective interaction should be repulsive, not attractive. From this sense, the phase separation we predicted in Ref. 9 could not occur in doped manganites. In fact, both the phase separation and the Wigner lattice were observed in the family of samples with different dopings. For example the phase separation was observed in $\mathrm{La}_{1-x} \mathrm{Ca}_{x} \mathrm{MnO}_{3}$ with $x=0.05$ and $0.08 .{ }^{20}$ It is worth pointing out that the electronic model is a simplified model for doped manganites since the degeneracy of $e_{g}$ electrons and the Jahn-Teller effect have been neglected. The importance of the orbital degeneracy of the $e_{g}$ electron has been extensively discussed, especially for the ferromagnetism near $x$ $=0$. If we take into account the orbital degeneracy, there may exist a superexchange virtual process in the ferromagnetic or A-type antiferromagnetic background, in which the superexchange coupling between different orbits instead of the spin indices in Fig. 1 could produce an attractive interaction, as we predicted in Ref. 9. The mechanism for phase separation may still be responsible for the experimental observation. The investigation along this direction is in progress. 
Before ending this paper, we would like to address the stability of the Wigner lattice with respect to the transfer $t$. Some experimental analysis suggested that a relatively small $t$ would favor forming the Wigner lattice, ${ }^{17}$ which seems to be in contradiction with the phase diagram in Fig. 2. In the present theory, the Wigner lattice occurs in a moderate value of $t$. On one hand, a large $t\left(\gg j_{h}\right)$, of course, will lead to the instability of the Wigner lattice and destroy the doubleexchange ferromagnetism. In that case, a paramagnetic phase should be favored at low temperatures. The perturbation technique used in this paper is also not valid. So the region of the Wigner lattice in Fig. 2 cannot be naively extended to the large $t$ case. On the other hand, when $t$ becomes very small compared with $j_{h}$, the Wigner lattice should also be unstable since a small $t$ means to enhance the ratio $j_{h} / t$ and a larger ratio is favorable to double-exchange ferromagnetism. If the antiferromagnetism from $t_{2 g}$ electrons could compete over the double-exchange ferromagnetism at $x$ $=0.5$, it would suppress ferromagnetism in all the ranges of

*Author to whom correspondence should be addressed. Electronic address: zwang@hkucc.hku.hk

${ }^{1}$ C. Zener, Phys. Rev. 82, 403 (1951); P.W. Anderson and H. Hasegawa, ibid. 100, 675 (1955).

${ }^{2}$ See Z. Jirak et al., J. Magn. Magn. Mater. 53, 153 (1985); K. Knizek et al., J. Solid State Chem. 100, 292 (1992); C.H. Chen et al., Phys. Rev. Lett. 71, 2461 (1993); P.G. Radaelli et al., ibid. 75, 4488 (1995); H. Kuwahara et al., Science 270, 961 (1995).

${ }^{3}$ Y. Tomioka, A. Asamitsu, Y. Moritomo, H. Kawahara, and Y. Tokura, Phys. Rev. Lett. 74, 5108 (1995).

${ }^{4}$ A.J. Millis, P.B. Littlewood, and B.I. Shraiman, Phys. Rev. Lett. 74, 5144 (1995); H. Röder, J. Zhang, and A.R. Bishop, ibid. 76, 1356 (1996); N. Furukawa, J. Phys. Soc. Jpn. 63, 3214 (1994); C.M. Varma, Phys. Rev. B 54, 7328 (1996); L. Sheng, D.Y. Xing, D.N. Sheng, and C.S. Ting, Phys. Rev. Lett. 79, 1710 (1997); S.Q. Shen and Z.D. Wang, Phys. Rev. B 59, 329 (1999).

${ }^{5}$ J. Inoue and S. Maekawa, Phys. Rev. Lett. 74, 3407 (1995).

${ }^{6}$ K. Kubo and N. Ohata, J. Phys. Soc. Jpn. 33, 21 (1972).

${ }^{7}$ We neglect a three-site term which describes the indirect hopping between the next-nearest-neighbor sites and is quite smaller than the direct hopping term.

${ }^{8}$ S.Q. Shen, Phys. Lett. A 235, 403 (1997).

${ }^{9}$ S.Q. Shen and Z.D. Wang, Phys. Rev. B 58, R8877 (1998). $x{ }^{8}$ The effective transfer $t \cos (\Theta / 2)$ is determined by either $t$ or $\Theta$ the angle of the two spins. The Wigner lattice is also accompanied by the strong antiferromagnetic correlation. The field-induced melting effect indicates that the Wigner lattice is unstable in the ferromagnetic background, which also indicates the important role of the antiferromagnetic correlation to stabilize the Wigner lattice. A smaller $j_{a f}$ will reduce the angle $\Theta$ and should also lead to the instability of the Wigner lattice. Thus, a small $t$ does not always favor forming the Wigner lattice.

In short, we derived an effective Hamiltonian for an extended Kondo lattice model, based on which a physical mechanism for charge ordering in half-doped manganites is naturally put forward.

\section{ACKNOWLEDGMENT}

This work was supported by a CRCG research grant at the University of Hong Kong.

${ }^{10}$ E. Dagotto et al., Phys. Rev. B 58, 6414 (1998); S. Yunoki et al., Phys. Rev. Lett. 80, 845 (1998).

${ }^{11}$ J. Riera, K. Hallberg, and E. Dagotto, Phys. Rev. Lett. 79, 713 (1997); E.L. Nagaev, Physica B 230-232, 816 (1997); L.J. Zou, Q.Q. Zheng, and H.Q. Lin, Phys. Rev. B 56, 13669 (1997); D.P. Arovas and F. Guinea, ibid. 58, 9150 (1998).

${ }^{12}$ S. Satpathy, Z.S. Popović, and F.R. Vukajlović, Phys. Rev. Lett. 76, 960 (1996); T. Mizokawa and A. Fujimori, Phys. Rev. B 54, 5368 (1996); L.F. Feiner and A.M. Oles, ibid. 59, 3295 (1999).

${ }^{13}$ Recent Monte Carlo simulations show that the Berry phase of the hopping amplitude has negligible effect in the value of the critical temperature [M.J. Calderon and L. Brey, Phys. Rev. B 58, 3286 (1998)]. If the electron path is not closed, we can neglect the Berry phase.

${ }^{14}$ E. Lieb and D. Mattis, J. Math. Phys. 3, 749 (1962).

${ }^{15}$ S.Q. Shen et al., Phys. Rev. Lett. 72, 1280 (1994); S.Q. Shen, Int. J. Mod. Phys. B 12, 709 (1998).

${ }^{16}$ E.O. Wollan et al., Phys. Rev. 100, 545 (1955); B.J. Sternlieb et al., Phys. Rev. Lett. 76, 2169 (1996).

${ }^{17}$ Y. Tokura et al., J. Appl. Phys. 79, 5288 (1996).

${ }^{18}$ J.D. Lee and B.I. Min, Phys. Rev. B 55, R14 713 (1997).

${ }^{19}$ S. Mori, C.H. Chen, and S.W. Cheong, Nature (London) 392, 473 (1998).

${ }^{20}$ G. Allodi et al., Phys. Rev. B 56, 6036 (1997); M. Hennion et al., Phys. Rev. Lett. 81, 1957 (1998). 